\title{
Vitamin D3 Supplementation Improved Cognitive Function in Diabetic Elderly Patients with Good Glycemic Control in Japan: A Pilot Study
}

Noboru Hasegawa ${ }^{{ }^{*}}$, Miyako Mochizuki ${ }^{2}$ and Takako Yamada ${ }^{3}$

${ }^{1}$ Department of Health and Medical Sciences, Ishikawa Prefectural Nursing University, 1-1 Gakuendai, Kahoku, Ishikawa 929-1210, Japan

${ }^{2}$ Kyoto Bunkyo Junior College, 80 Senzoku, Makishima-cho, Uji, Kyoto 611-0041, Japan

${ }^{3}$ Bukkyo University, 7, Higashitoganoo-cho, Nishinokyo, Nakagyo-ku, Kyoto604-8418, Japan

\section{Abstract}

Background: We studied the effects of vitamin D3 supplementation on serum levels of glycosylated hemoglobin, 25-hydroxyvitamin D and 1,25-hydroxyvitamin D, and on cognitive function in elderly diabetic patients.

Methods: We enrolled 6 male and 9 female Japanese patients (age: $74.4 \pm 6.2$ ) with controlled diabetes mellitus who attended an outpatient clinic. The serum glycosylated hemoglobin, 25-hydroxy vitamin D, and 1,25-hydroxy vitamin D concentrations were measured prior to supplementation and after 3, 9 and 12 months of supplementation with vitamin D3 (500 IU/day of vitamin D3, corresponding to twice the daily requirement). The Montreal Cognitive Assessment-Japanese version was used for the cognitive function test. Data were analyzed using a two-way ANOVA or the Friedman test followed by a Bartlett analysis for homoscedasticity.

Results: The results showed that before vitamin D3 supplementation 25-hydroxy vitamin D level was < 20ng/ $\mathrm{mL}$ (deficient) in all patients except one (insufficient). Our results show that individual 25-hydroxy vitamin $\mathrm{D}$ levels vary significantly from each other and by supplementation duration. The vitamin $\mathrm{D}$ deficient group decreased from 14 to 8 patients (5: became insufficient, 1: became sufficient) and the insufficient group decreased from 1 to 0patients after 9 months. The 1,25-hydroxyvitamin D and glycosylated hemoglobin levels did not vary significantly with duration of supplementation. The Montreal Cognitive AssessmentJapanese version scores did not vary significantly from each other or by supplementation duration. However, we observed increased Montreal Cognitive Assessment-Japanese version scores after supplementation in $60 \%$ of the patients.

Conclusion: These findings show that vitamin D supplementation is associated with improved serum vitamin $\mathrm{D}$ levels and might improve cognitive function.

\section{Introduction}

Diabetes mellitus (DM) is characterized by chronic hyperglycemia caused by impaired insulin secretion, peripheral insulin resistance, or both [1].

Type $2 \mathrm{DM}$ has been shown to increase the risk for cognitive function [2,3]. People with DM have been shown to have a lower performance on the Mini-Mental State Examination (MMSE) [4].

Vitamin D is a secosteroid associated with peripheral calcium homeostasis and nervous system function [5]. Vitamin D is available in two forms, vitamin D2 from plants and D3 from animals. Both vitamin D2 and D3 are biologically inert and require activation through two hydroxylation processes involving 25-hydrooxylase (CYP2R1) and 1a-hydroxylase (CYP27B1), located in the liver and kidney, respectively [6]. 1, 25-dihydroxyvitamin D $(1,25 \mathrm{OHD})$ is a biologically active metabolite produced by two hydroxylation reaction steps in the nervous system [7].

It has been suggested that vitamin $\mathrm{D}$ reduces the risk of type 2 diabetes. In one cohort study, the intake of vitamin D supplements was inversely associated with the development of type 2 diabetes [8]. However, prospective studies have not found an association between 25OHD concentration and cognitive function in DM patients.

Recent evidence has identified a beneficial role of vitamin D in maintaining cognitive functions [9]. Vitamin D receptors and CYP27B1 have been found in the brain (hippocampus) [10]. Low patients in Japan. hsgwn@ishikawa-nu.ac.jp https://doi.org/10.15344/2394-4978/2019/311 original author and source are credited.

\section{Publication History:}

Received: June 17, 2019

Accepted: July 27, 2019

Published: July 29, 2019

\section{Keywords:}

Vitamin D, Diabetes Mellitus, Cognitive function, Chronic hyperglycemia greater risk of cognitive impairment in older as well as younger adults using the Montreal Cognitive Assessment (MoCA) Arabic version [11]. Vitamin D supplementation caused significant improvement in cognitive performance assessed by MMSE score in Alzheimer's disease [12-14]. In our latest study, a significant positive correlation was found between urinary 25-hydroxy vitamin D (25OHD)/creatinine and MMSE or the Montreal Cognitive Assessment-Japanese version the Montreal Cognitive Assessment-Japanese version (MoCA-J) [15].

There are no data suggesting that vitamin D supplementation may be associated with improved cognitive function in DM patients. Therefore, the present study was designed to investigate the effect of vitamin D3 supplementation corresponding to twice the daily requirement for 9 months on cognitive impairment in elderly diabetic

*Corresponding Author: Prof. Noboru Hasegawa, Department of Health and Medical Sciences, Ishikawa Prefectural Nursing University, 1-1 Gakuendai, Kahoku, Ishikawa 929-1210, Japan, Tel: +81-76-281-8300, Fax: +81-281-8319; E-mail:

Citation: Hasegawa N, Mochizuki M, Yamada T (2019) Vitamin D3 Supplementation Improved Cognitive Function in Diabetic Elderly Patients with Good Glycemic Control in Japan: A Pilot Study. Int J Nurs Clin Pract 6: 311. doi:

Copyright: (C) 2019 Hasegawa et al. This is an open-access article distributed under the terms of the Creative Commons Attribution License, which permits unrestricted use, distribution, and reproduction in any medium, provided the 
Citation: Hasegawa N, Mochizuki M, Yamada T (2019) Vitamin D3 Supplementation Improved Cognitive Function in Diabetic Elderly Patients with Good Glycemic Control in Japan: A Pilot Study. Int J Nurs Clin Pract 6: 311. doi: https://doi.org/10.15344/2394-4978/2019/311

Page 2 of 4

\section{Materials and Methods}

\section{Subjects and Setting}

Prior to the study, approval was obtained from the ethics committee of Kyoto Bunkyo Junior College (project registration number in 2016: 7) and Aichi Medical College for Physical and Occupational Therapy (Project registration number in 2016: 468). We enrolled 6 male and 9 female Japanese patients (age: $74.4 \pm 6.2$ ) with controlled diabetes mellitus who attended an outpatient clinic. The researchers were present at the clinic to assure the proper management of safety and confidentiality in the study. The manager of the clinic invited patients to participate in the study, and all the subjects whose participation was requested from September in 2018 to May in 2019 were selected. All subjects took vitamin D supplements (500 IU/day of vitamin D3 purchased from UHA Mikakuto Co., Ltd., Osaka) for 9 months.

\section{Cognitive function test}

MoCA-Jmay be better at detecting early cognitive dysfunction [16] and was used for the cognitive function test. The maximum score for the MoCA-J is 30 points and individuals with a score of 26 points the recommended [12]. The MoCA-Jmay be better at detecting early cognitive dysfunction [16] and was used for the cognitive function test in DM patients [17]. The test was performed by verbal questioning of 5- to 10-min duration by skilled occupational and physical therapists.

\section{Serum 1, 25OHD, 25OHD and HbA1c Assay}

Blood was collected by venipuncture and serum 1,25OHD, 25OHD and $\mathrm{HbAlc}$ concentrations were measured bySRL Inc.(Osaka, Japan).

\section{Statistical analysis}

The differences between before and after intervention with vitamin D3 supplements were evaluated using two-way ANOVA or the Friedman test followed by Bartlett analysis for homoscedasticity. A p-value of $<0.05$ was considered to be statistically significant. Analyses were carried out using SPSS 21 for Windows (IBM, Japan).

\section{Results and Discussion}

\section{Study subjects}

Characteristics of the study subjects are shown in Table 1. Mean age was 71.7 years for males $(n=6)$ and 76.2 years for females $(n=9)$. Mean $\mathrm{HbAlc}$ was $6.3 \%$ for males and $6.9 \%$ for females. These values indicated good glycemic control. Obesity was defined as a body-mass index (BMI) of $\geq 25.0 \mathrm{~kg} / \mathrm{m}^{2}$. The prevalence of obesity defined by BMI was $16.6 \%$ in males and $44.4 \%$ in females.

\begin{tabular}{|l|l|}
\hline No. of participants (\% male) & $15(33.3)$ \\
\hline Age $(\mathrm{y})$ & $74.4 \pm 6.2$ \\
\hline Body height $(\mathrm{cm})$ & $158.6 \pm 8.3$ \\
\hline Body weight $(\mathrm{kg})$ & $61.6 \pm 7.3$ \\
\hline BMI $\left(\mathrm{kg} / \mathrm{m}^{2}\right)$ & $24.5 \pm 2.7$ \\
\hline Table1: Characteristics of the study subjects.
\end{tabular}

\section{Change in serum 25OHD, 1, 25OHD and HbAlc}

Serum 25OHD was classified as normal $(>=30 \mathrm{ng} / \mathrm{ml})$, insufficient ( $>20$ to 29.9 ), or deficient $(<=20)$. In this study, the level was deficient in all except one patient (insufficient). The results from a two-way ANOVA showed that individual 25OHD levels varied significantly from each other $(\mathrm{P}=0.004)$ and by supplementation duration $(\mathrm{P}=$ 0.002) (Figure 1). Three month intake of vitamin D3 supplements increased serum 25OHD concentration significantly $(p=0.04)$ and 9 month intake increased serum 25OHD within the insufficiency and sufficiency levels ( 5 subjects: $>20 \mathrm{ng} / \mathrm{mL}$ and $<30 \mathrm{ng} / \mathrm{mL}, 1$ subject: $>30 \mathrm{ng} / \mathrm{mL}$ ). These results suggest that vitamin D3 supplement intake maintained serum 25OHD levels. A multiple linear regression study revealed an inverse significant association between $\mathrm{HbAlc}$ and 25OHD levels [18]. These results suggest that screening for vitamin D status may be beneficial in DM patients.

Serum HbAlc and 1, 25OHD did not significantly by supplementation duration in the Friedman test. This was assumed to be because all participants had their HbAlc levels checked once a month and was taking medications for treatment of DM.

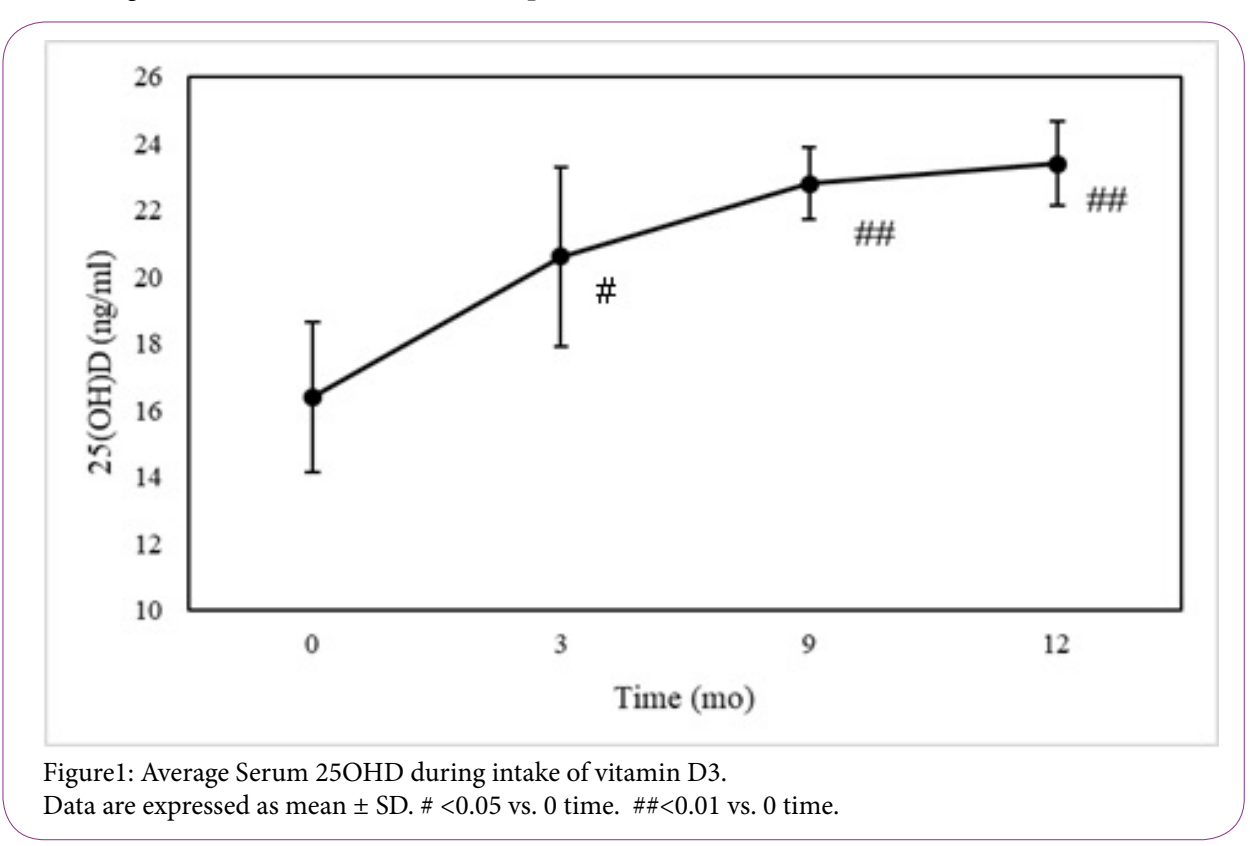


Citation: Hasegawa N, Mochizuki M, Yamada T (2019) Vitamin D3 Supplementation Improved Cognitive Function in Diabetic Elderly Patients with Good Glycemic Control in Japan: A Pilot Study. Int J Nurs Clin Pract 6: 311. doi: https://doi.org/10.15344/2394-4978/2019/311

\section{Cognitive function}

MoCA-J did not vary significantly between individuals $(\mathrm{P}=0.12)$ or by supplementation duration $(\mathrm{P}=0.79)$ in a two-way ANOVA. However, we observed an increase in MoCA-J score after supplementation in $60 \%$ of the patients (Figure 2).

Many epidemiological studies have shown that diabetes is a risk factor for cognitive impairment. Recent evidence suggests a potential beneficial role of vitamin $\mathrm{D}$ in maintaining cognitive function [8]. In the present study, daily supplementation of 500IU vitamin D3 (serum $25 \mathrm{OHD} \leq 30 \mathrm{ng} / \mathrm{mL}$ ) was associated with a change in cognitive function.

Low vitamin $\mathrm{D}$ has been associated with the risk of developing Alzheimer's disease $[12,14]$. In the current study, dementia patients treated with vitamin D for 6 months improved their performance on a cognition test (MMSE score) [19]. Over expression of reactive oxygen species (ROS) stimulated by disruption of cerebral blood flow was shown to be one of the main causes of vascular dementia-induced cognitive deficits in a rat model [20]. 1, 25OHD was reported to have significant physiological antioxidant activity [21]. These results suggest a potential role of vitamin $\mathrm{D}$ in treating patients with cognitive dysfunction in DM.

Further study of optimal 25OHD levels for maintaining physical and cognitive functions and preventing falls in DM patients is needed.

\section{Conclusion}

These findings indicate that vitamin D supplementation might improve cognitive function.

\section{Author Contributions}

Dr. Hasegawa was responsible for the study conception, design, and analysis, interpretation of data, and drafting of the manuscript.

Ms. Mochizuki was responsible for data acquisition and proof reading of the manuscript, and she participated in the data analysis.

Dr. Yamada was responsible for the data acquisition and proof reading of the manuscript.

\section{Acknowledgements}

The author acknowledges Dr. Yasuaki Fukumoto and Michiko Iwata for then kind help.

\section{Funding}

This work was supported by JSPS KAKENHI Grant Number JP22500682.

\section{Competing Interest}

The authors declare that they have no competing interest.

\section{References}

1. Ndisang JF, Vannacci A, Rastogi S (2017) Insulin resistance, type 1 and type 2 diabetes, and related complications 2017. J Diabetes Res.

2. Hanyu H (2019) Diabetes-related dementia. Adv Exp Med Biol 1128: 147160.

3. Kim HJ, An SY, Han SJ, Kim DJ, Hong CH, et al. (2019) The association of diabetes duration and glycemic control with depression in elderly men with type 2 diabetes mellitus. J Res Med Sci 24: 17.

4. Xiu S, Zheng Z, Liao Q, Chan P (2019) Different risk factors for cognitive impairment among community-dwelling elderly, with impaired fasting glucose or diabetes. Diabetes Metab Syndr Obes 12: 121-130.

5. DeLuca GC, Kimball SM, Kolasinski J, Ramagopalan SV, Ebers GC, et al. (2013) Review: the role of vitamin D in nervous system health and disease. Neuropathol Appl Neurobiol 39: 458-484.

6. Sarfraz Z (2015) Power of Vitamin D (3rd edition): A Vitamin D Book that Contains the Most Scientific, Useful and Practical Information About Vitamin D - Hormone D. Create Space Independent Publishing Platform, USA.

7. Garcion E, Wion-Barbot N, Montero-Menei CN, Berger F, Wion D, et al. (2002) New clues about vitamin D functions in the nervous System. Trends Endocrinol Metab 13: 100-105.

8. Mattila C, knekt P, Männistö S, Rissanen H, Laaksonen MA, et al. (2007) Serum 25-hydroxyvitamin $D$ concentration and subsequent risk of type 2 diabetes. Diabetes Care 30: 2569-2570.

9. Houston DK (2015) Vitamin D and age-related health outcomes: Movement, Mood, and Memory. Curr Nutr Rep 4: 185-200.

10. Eyles DW, Smith S, kinobe R, Hewison M, McGrath JJ, et al. (2005) Distribution of the vitamin $\mathrm{D}$ receptor and 1 alpha-hydroxylase in humanbrain. J Chem Neuroanat 29: 21-30.

11. Darwish $H$, Zeinoun $P$, Ghusn $H$, Khoury $B$, Tamim $H$, et al. (2015) Serum 25-Hydroxyvitamin $D$ predicts cognitive performance in adults. Neuropsychiatr Dis Treat 11: 2217-2223.

12. Gangwar AK, Rawat A, Tiwari S, Tiwari SC, Narayan J, et al. (2015) Role of Vitamin-D in the prevention and treatment of Alzheimer's disease. Indian JPhysiol Pharmacol 59: 94-99. 
Citation: Hasegawa N, Mochizuki M, Yamada T (2019) Vitamin D3 Supplementation Improved Cognitive Function in Diabetic Elderly Patients with Good Glycemic Control in Japan: A Pilot Study. Int J Nurs Clin Pract 6: 311. doi: https://doi.org/10.15344/2394-4978/2019/311

13. Wang F, Luo J, Ding D, Zhao Q, Guo Q, et al. (2019) Elevated fasting blood glucose level increases the risk of cognitive decline among older adults with diabetes mellitus: the Shanghai aging study. J Alzheimers Dis 67: 12551265.

14. Balion C, Griffith LE, Strifler L, Henderson M, Patterson C, et al. (2012) Vitamin $D$, cognition, and dementia: a systematic review and meta-analysis. Neurology 79: 1397-1405.

15. Hasegawa N, Mochizuki M, Kato M, Yamada T, Shimizu N, et al. (2016) Serum 1,25-Hydroxyvitamin D: auseful index of cognitive and physical functional impairment in healthy older adults in Japan: A pilot study. Health 8: 1679-1686.

16. Nasreddine ZS, Phillips NA, Bédirian V, Charbonneau S, Whitehead V, et al (2005) The Montreal Cognitive Assessment, MoCA: a brief screening tool for mild cognitive impairment. J Am Geriatr Soc 53: 695-699.

17. Alagiakrishnan K, Zhao N, Mereu L, Senior P, Senthilselvan A, et al. (2013) Montreal cognitive assessment is superior to Standardized Mini-Mental Status Exam in detecting mild cognitive impairment in the middle-aged and elderly patients with type 2 diabetes mellitus. Biomed Res Int 186106: 1-5.

18. Alkhatatbeh MJ, Abdul-Razzak KK (2018) Association between serum 25-hydroxyvitamin $D$, hemoglobin $A 1 c$ and fasting blood glucose levels in adults with diabetes mellitus. Biomed Rep 9: 523-530.

19. Annweiler C, Herrmann FR, Fantino B, Brugg B, Beauchet O, et al. (2012) Effectiveness of the combination of memantine plus vitamin D on cognition in patients with Alzheimer disease: a pre-post pilot study. Cogn Behav Neurol 25: 121-127.

20. Li Z, Wang Y, Xie Y, Yang Z, Zhang T, et al. (2011) Protective Effects of exogenous hydrogen sulfide on neurons of hippocampus in a rat model of brain ischemia. Neurochem Res 36: 1840-1849.

21. Calgaroto NS, Thomé GR, da Costa P, Baldissareli J, Hussein FA, et al. (2014) Effect of Vitamin D3 on behavioural and biochemical parameters in diabetes type 1-inducedrats. Cell Biochem Funct 32: 502-510. 